\title{
Nonlinear stochastic relaxation dynamics in spin-crossover solid-state compounds
}

\author{
Iu.V. Gudyma, A.Iu. Maksymov \\ Chernivtsi National University, Department of General Physics, 58012 Chernivtsi, Ukraine, \\ E-mail:yugudyma@gmail.com
}

\begin{abstract}
A study of dynamic of spin-crossover solid-state compound has been carried out. The investigated macroscopic phenomenological model for molecular spin-crossover complexes with optical control parameter has been extended to the case of noise action. The noise-driven phase transition was observed. Also, ascertained was the role of additive noise as a main factor for suppressing the potential barrier height.
\end{abstract}

Keywords: optical bistability, additive noise, multiplicative noise, noise-driven dynamics.

Manuscript received 10.10.10; accepted for publication 02.12.10; published online 30.12.10.

\section{Introduction}

With increasing needs in computing enormous volume of information, investigation of new molecular materials which may satisfy the requirements in the field of informational technology becomes actual. One of the most promising bistable materials is spin-crossover coordination complexes that possess two stable states: paramagnetic high-spin (HS) and diamagnetic low-spin (LS) states [1]. There is a class of inorganic complexes of chemical elements with $d^{4}-d^{7}$ electron configuration and central transition metal ion located in octahedral ligand field. Under the influence of environmental perturbation, namely: light irradiation, magnetic field, temperature, noise influence and others, there possible is the transition between the states of spincrossover compounds. The transition provokes a drastic change in properties of spin-crossover complexes, in particular, magnetic properties due to spin rearrangement between sublevels of $3 d$-orbitals. Thus, there exist many experimental works where bistability in magnetic susceptibility is shown [2].

One of the most important developments in researches of spin crossover was that the equilibrium existing between high-spin and low-spin species could be perturbed by laser irradiation. A light irradiation of a spin-crossover system in the solid state at low temperature induces partial or complete conversion of a low-spin state to a high-spin metastable one with virtually infinitely long lifetime. This solid state effect became known as the LIESST effect (light induced excited spin state trapping) [3]. This effect is the basis of different light-induced bistability effects that provoke appearance of optical (light induced optical hysteresis, shortly $\mathrm{LIOH}$ ) and thermal hysteresis (light induced thermal hysteresis, shortly LITH). A light-induced bistability in a spin transition solids leading to thermal and optical hysteresis has been well studied in $[4,5]$.

Until now, many experiments were performed to study the process and physics of the transition from one state to another, but there is still no clarity in this problem. Here, we propose to represent the model of spin-crossover complexes as a macroscopic phenomenological model written in terms of relaxation rate with light-induced phase transition. To fuller satisfy the condition of physical reality, we have taken into account environmental action on the system dynamics as additive and multiplicative Gaussian distributed noises.

The environmental random noise influencing on spin-crossover solids can be represented as an interaction with a heat bath. This model may be described by macroscopic kinetic stochastic differential equation with a deterministic term possessing slow degree of freedom and random term with fast degree of freedom. Dynamics of a system being in contact with an environment playing a role of the heat bath is based on the concept of the Langevin equation. Such assumption is possible because the condition of heat bath does not depend on spin configuration. Detailed theoretical analysis of the states of spin-crossover compounds under cross-correlated noises was made in [6].

On the assumption of possible technological usage of these materials as data storage systems or display systems, it is important to know the lifetime of 
metastable state or transition time from one state to another. Due to environmental perturbation and size limitation in any real system, the lifetime of a metastable state is not infinitely long. In this work, we also estimated the time of decay of the metastable state in a mean field approach by defining the mean first passage time (MFPT) for this metastable state. The estimation of MFPT was made in Kramers-like approximation by using a reflecting boundary condition at the minimum of the metastable state and absorption boundary condition at maximum of the potential barrier height that separates the stable and metastable states. A simple but very general approach to the classical MFPT problem was offered by Pontryagin et al. [7], which has been extended by us for investigation MFPT of spin-crossover solids.

\section{Model}

Here we regard the macroscopic phenomenological model of spin-crossover compounds in terms of a relaxation rate in the mean field approach. With this aim, it is necessary to admit one of the states of spincrossover compound as a metastable state, and the other as a stable one. In our investigation, we did not focused on specific spin-crossover compound, but due to more widespread spin-crossover compounds with LS state as a ground one, we have considered that paramagnetic HS state is metastable. For the HS fractions $n_{H}$, the static relaxation equation first investigated by Hauser with the Mainz group [8-10] is as follows:

$k_{H L}\left(n_{H}, T\right)=k_{\infty} \exp \left[-\frac{E_{a}\left(n_{H}\right)}{k_{B} T}\right]$,

with

$E_{a}\left(n_{H}\right)=E_{a}(0)+\alpha(T) k_{B} T n_{H}$,

where $k_{\infty}$ is the high-temperature asymptotic of the relaxation rates at the end of the process $\left(n_{H} \rightarrow 0\right)$; $E_{a}(0)$ is the activation energy; $k_{B}$ is the Boltzmann constant; $T$ is the temperature, and $\alpha(T)$ is the selfacceleration factor of relaxation defined by the cooperative coefficient and depending linearly on the relative atomic concentration of the spin-crossover element and is proportional to the inverse temperature [11]. For simplicity, the activation energy has been equaled to zero, so the relaxation equation (1) takes the form:

$k_{H L}\left(n_{H}, T\right)=k_{\infty} \exp \left[-\alpha(T) n_{H}\right]$.

The general form of macroscopic master equation in condition of light irradiation can be expressed in terms of photoexcitation and relaxation high-spin fraction flows from HS to LS state and vice versa [4]:

$\frac{d n_{H}}{d t}=\Phi_{e x c}-\Phi_{r e l}$.
The form of photoexcitation $\Phi_{\text {exc }}$ and relaxation $\Phi_{\text {rel }} n_{H}$-flows is as follows:

$\Phi_{\text {exc }}=I_{0} \omega\left(1-n_{H}\right)$,

$\Phi_{\text {rel }}=n_{H} k_{\infty} \exp \left(-\alpha n_{H}\right)$,

where $I_{0} \omega$ is the probability per time unit for a LS molecule to switch to the HS state, with the beam intensity $I_{0}$ and a proportionality factor $\omega$ that includes the absorption cross-section of the optically active element (here the bulk absorbance is neglected). By introducing a new variable $\tau=t k_{\infty}$ and taking into account a denotation $\beta=I_{0} \omega / k_{\infty}$, it is easy to obtain the final macroscopic master equation of the described model with non-dimensional quantities:

$\frac{d n_{H}}{d \tau}=\beta\left(1-n_{H}\right)-n_{H} \exp \left(-\alpha n_{H}\right)$.

From the stationary condition of the system when $\Phi_{\text {exc }}=\Phi_{\text {rel }}$, which is realized at the spin-crossover point, one can estimate the possibility of existence of all theoretical realizable stable and unstable states. A comprehensive analysis of static and dynamical nonequilibrium kinetics of spin-crossover compounds was made by Gudyma in [12] and Varret in [13].

\section{Macroscopic stationary dynamics of spin-crossover compounds}

Any real system being in contact with environment may be modeled as a system that interacts with a heath bath. To more accurately describe our model, we must take into consideration an environmental noise influence. In other words, the control parameter $\beta$ from Eq. (6) is not constant, but fluctuates in time. Besides, it is necessary to take into account the direct action of heat bath on the system. Thus, it is need to assume that $\beta(t)=\beta+\xi(t)$ in Eq. (6), which gives the following stochastic differential equation in Stratonovich interpretation:

$$
\begin{aligned}
& \frac{d n_{H}}{d \tau}=\Gamma\left(1-n_{H}\right)-n_{H} \exp \left(-\alpha n_{H}\right)+ \\
& +\left(1-n_{H}\right) \xi(\tau)+v(\tau)=f\left(n_{H}\right)+g\left(n_{H}\right) \xi(\tau)+v(\tau),
\end{aligned}
$$

where the noise correlation functions are:

$$
\begin{aligned}
& \left\langle\Gamma(\tau) \xi\left(\tau^{\prime}\right)\right\rangle=2 \sigma^{2} \delta\left(\tau^{\prime}-\tau\right), \\
& \left\langle v(\tau) v\left(\tau^{\prime}\right)\right\rangle=2 \varepsilon^{2} \delta\left(\tau^{\prime}-\tau\right), \\
& \left\langle\xi(\tau) v\left(\tau^{\prime}\right)\right\rangle=2 \chi \sigma \varepsilon \delta\left(\tau^{\prime}-\tau\right),
\end{aligned}
$$

where $\sigma$ and $\varepsilon$ are the intensity of multiplicative noise and additive noise, respectively, which correspond to the influence of internal and external fluctuation on the system; $\chi$ is the correlation coefficient between additive and multiplicative noises. In a general case, external 
environmental fluctuations can influence on the internal fluctuations but, for simplicity, we did not take this effect into account. The analysis of states in spincrossover solids under cross-correlated noises was made in the paper [6], which is based on the mathematical tools developed in [14].

The Eq. (7) can be reduced to the equivalent stochastic differential equation with one effective multiplicative noise [16]:

$$
\frac{d n_{H}}{d \tau}=f\left(n_{H}\right)+G\left(n_{H}\right) \Xi(\tau)
$$

with the condition

$$
\left\langle\Xi(\tau) \Xi\left(\tau^{\prime}\right)\right\rangle=2 \delta\left(\tau^{\prime}-\tau\right) .
$$

The effective noise amplitude $G\left(n_{H}\right)$ is determined as follows:

$$
G\left(n_{H}\right)=\sqrt{\sigma^{2}\left(1-n_{H}\right)^{2}+2 \chi \sigma \varepsilon\left(1-n_{H}\right)+\varepsilon^{2}}
$$

For nonlinear stochastic differential equations, one can obtain the Fokker-Planck equation corresponding to the Langevin equation in the following form [15]:

$$
\frac{\partial P\left(n_{H}, \tau\right)}{\partial \tau}=\frac{\partial}{\partial n_{H}} f\left(n_{H}\right) P\left(n_{H}\right)+\frac{1}{2} \frac{\partial^{2}}{\partial n_{H}^{2}} G\left(n_{H}\right) P\left(n_{H}\right) .
$$

By solving the Fokker-Planck equation (12), we found the probability of system location at the phase space point. The stationary solution of this equation has the Gibbs-Boltzmann form:

$$
P_{s t}\left(n_{H}\right)=N \exp \left(-U_{e f f}\right),
$$

where $N$ is the normalization constant and

$$
U_{\text {eff }}=-\int_{0}^{n_{H}} \frac{f\left(n_{H}\right)-G\left(n_{H}\right) \frac{\partial}{\partial n_{H}} G\left(n_{H}\right)}{\left[G\left(n_{H}\right)\right]^{2}}
$$

represents the effective dynamical system potential. The extremes of this potential correspond to stationary fixed points of system dynamics. Bistable range of system with corresponding dynamic potentials at spinodal points obtained from Eq. (6) with stationary equilibrium condition and Eq. (14) are consequently shown in Fig. 1.

The intersection point between the curves $\Phi_{\text {exc }}$ (photoexcited $n_{H}$-flow marked as dotted curve in the plots) and $\Phi_{\text {rel }}$ (relaxed $n_{H}$-flow marked as a dashed curve) from Fig. 1 represent the possible steady and unsteady states, thus we defined the range of bistability. The effective potential $U_{\text {eff }}$ (marked as a solid curve on all plots from Fig. 1) at the spinodal points (the critical points of phase transition) between monostable-bistable range and bistable-monostable range are reflected in Fig. 1a and Fig. 1c, consequently. Shown in this figure is light-induced optical hysteresis (LIOH) where light irradiation in the first approach is the main control parameter. Analysis showed that noise acting also may lead to transition from one state to another. Nonstationary overall studies of noise action on the system are described in the following section.
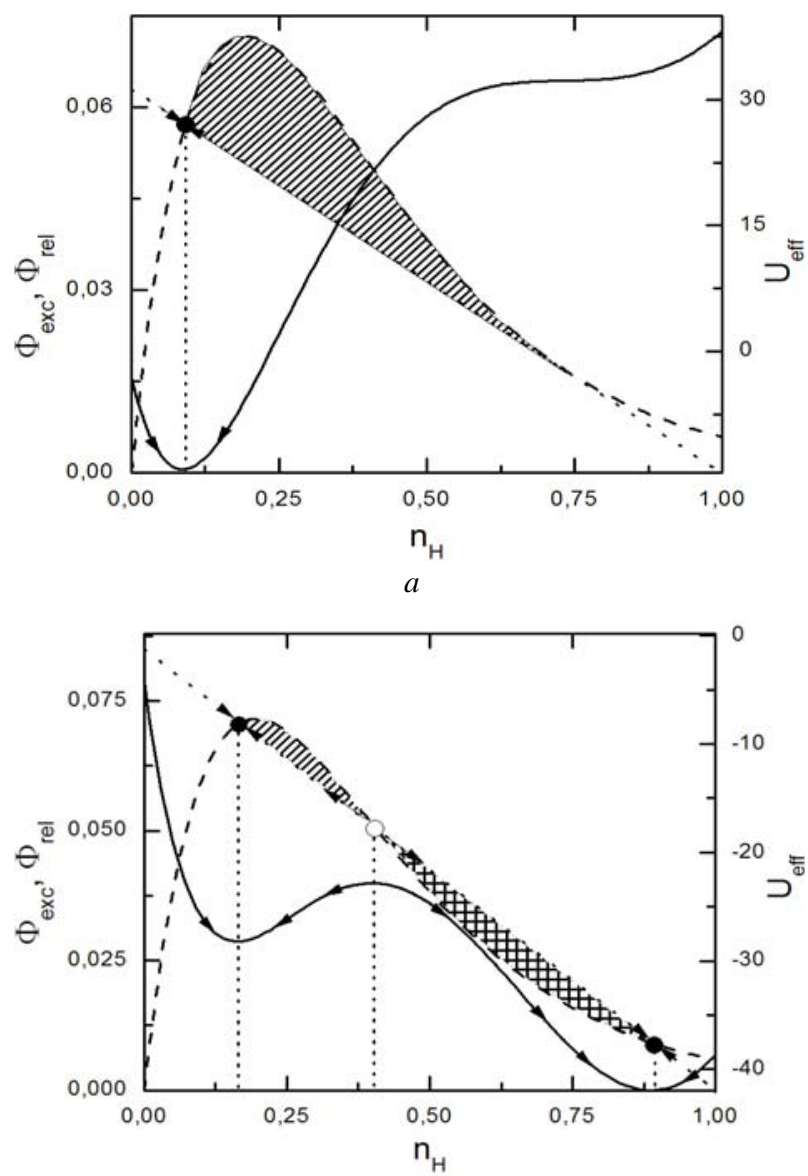

$b$

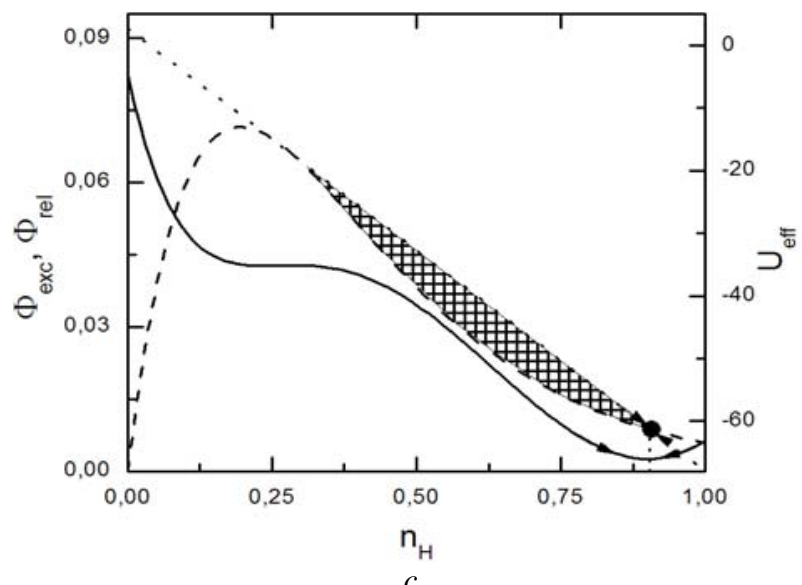

Fig. 1. Light-induced bistable range with the effective dynamical potential $U_{\text {eff }}$ : (a), (c) indicate the spinodal points of one state - two states transition; (b) is the realization of bistable state. 


\section{Noise driven dynamics of spin-crossover compounds}

It is generally known that the more suitable to find solution of numerically ordinary differential equations similar to Eq. (6) are the second-order Runge-Kutt type methods. One of the main requirements in this case is that the functions appearing in the equation are differentiable to some required order. But, unfortunately, if using the white-noise functions as in Eqs (7) and (8), even for a single realization of the white-noise term, these functions are highly irregular and nondifferentiable. So, the only non-rigorous approach via a series of delta-functions spread all over the real axis can be realized. To solve the presented stochastic differential equation in the non-stationary case, we need to use more powerful computational methods. These methods may be developed from the second-order Runge-Kutt method with suitable modification based on application of integral algorithms described in [17]. It results in the Milshtein method:

$n_{H}\left(\tau^{\prime}\right)=n_{H}(\tau)+h^{1 / 2} G\left(n_{H}(\tau)\right) u(\tau)+$

$+h\left[f\left(n_{H}(\tau)\right)+\frac{1}{2} G\left(n_{H}(\tau)\right) \frac{\partial}{\partial n_{H}}\left\{G\left(n_{H}(\tau)\right)\right\}(u(\tau))^{2}\right]+$ $+O\left[h^{3 / 2}\right]$

where $h^{1 / 2} u(\tau)=\int_{\tau}^{\tau^{\prime}} \Xi(s) d s ; h$ is the order of a time step in the system dynamics.

We got the non-stationary solution of Eq. (7) from explicit two-stage second-order Runge-Kutt method well known as the Heun or explicit trapezoidal method $[17,18]$ :

$y=h f\left(\tau, n_{H}(\tau)\right)+h^{1 / 2} u(\tau) G\left(\tau, n_{H}(\tau)\right)$,

$n_{H}\left(\tau^{\prime}\right)=n_{H}(\tau)+\frac{h}{2}\left[f\left(\tau, n_{H}(\tau)\right)+f\left(\tau^{\prime}, n_{H}(\tau)+y\right)\right]+$

$+\frac{h^{1 / 2} u(\tau)}{2}\left[G\left(\tau, n_{H}(\tau)\right)+G\left(\tau^{\prime}, n_{H}(\tau)+y\right)\right]$.

The Eq. (16) is obtained from the Euler equation with stochastic process in its turn derived from the Milshtein method (15), if $\frac{\partial}{\partial n_{H}}\left\{G\left(n_{H}(\tau)\right)\right\}=0$

corresponds to an additive noise case) [17]. The Euler equation resulting from (16) is as follows:

$$
n_{H}\left(\tau^{\prime}\right)=n_{H}(\tau)+h f\left(n_{H}(\tau)\right)+G\left(n_{H}(\tau)\right) h^{1 / 2} u(t)+O\left[h^{3 / 2}\right] .
$$

The derived Euler equation in midpoint quadratures has the following form:

$$
\begin{aligned}
& n_{H}\left(\tau^{\prime}\right)=n_{H}(\tau)+\frac{h}{2}\left[f\left(\tau, n_{H}(\tau)\right)+f\left(\tau^{\prime}, n_{H}\left(\tau^{\prime}\right)\right)\right]+ \\
& +\frac{h^{1 / 2} u(\tau)}{2}\left[G\left(\tau, n_{H}(\tau)\right)+G\left(\tau^{\prime}, n_{H}\left(\tau^{\prime}\right)\right)\right] .
\end{aligned}
$$

By replacing $n_{H}\left(\tau^{\prime}\right)$ on the right-handside in the later equation by the predictor of the Euler method (17) again, we arrive to the Heun equation (16). Trajectories from Eq. (16) represent the solution of Eq. (9) with stochastic fluctuation in time of HS fractions.

We carried out the simulation of HS fraction dynamic for 100 trajectories with 50000 time step and obtained the distribution of probability density functions (pdf) of HS fraction for a non-stationary case. Shown in Fig. 2 are the distributions of pdf in stationary and nonstationary cases under noise action. From the plots, we observe a qualitative difference in action of additive noise and that of multiplicative noise: while the increase in multiplicative noise intensity leads to the first-order phase transition, the increase in additive noise intensity only suppresses the potential barrier height, which makes HS and LS states to be unrecognized and is undesirable for sensor devices.

Shown in Fig. 3 are the examples of stochastic trajectories for values of additive noise intensity $\sigma=0.001$ and $\sigma=0.18$. The later value corresponds to spinodal point between bistable-monostable regions. The stochastic trajectories in Fig. 3 are only illustration of the processes that take place in a noise-driven system. In practice, it is impossible to determine the one isolated trajectory, due to time-scale of occurred processes. So, one can only detect the average value of stochastic fluctuations, which is important to know for designing stable spin-crossover sensor devices.

\section{Lifetime of the metastable state in the mean field approach}

As we have seen above, the dynamic of the HS fraction in spin-crossover compounds under environmental noise action is modeled as fluctuation in time of Brownian particle. After the pioneering contribution by Kramers, well described in [19], the escape problem of Brownian particle became an important subject of studies in nonlinear stochastic systems. The most common analysis of metastable state decay is based on the MFPT technique in a Kramer-like approximation described in general aspects by Pontryagin in [7]. The escape time from a metastable state in the stationary case was studied in $[12,20]$. The general expression for MFPT is as follows:

$T\left(a \rightarrow n_{H}\right)=\int_{n_{H}}^{b} \frac{d x}{\psi(x)} \int_{a}^{x} \frac{\psi(x)}{\left[G_{\text {rand }}^{e f f}(x)\right]^{2}}$,

where

$\psi(z)=\exp \left[\int_{a}^{z} \frac{2 f_{\text {det }}\left(z^{\prime}\right)}{\left[G_{\text {rand }}^{\text {eff }}\left(z^{\prime}\right)\right]^{2}} d z^{\prime}\right]$.

Here, we supplemented theoretically obtained MFPT by computing simulation. The results are shown 
in Fig. 4. Shown in the figure is estimation of MFPT between stationary and non-stationary cases (see the normalized time values), so the intersection of time curves is not necessary to take into consideration.
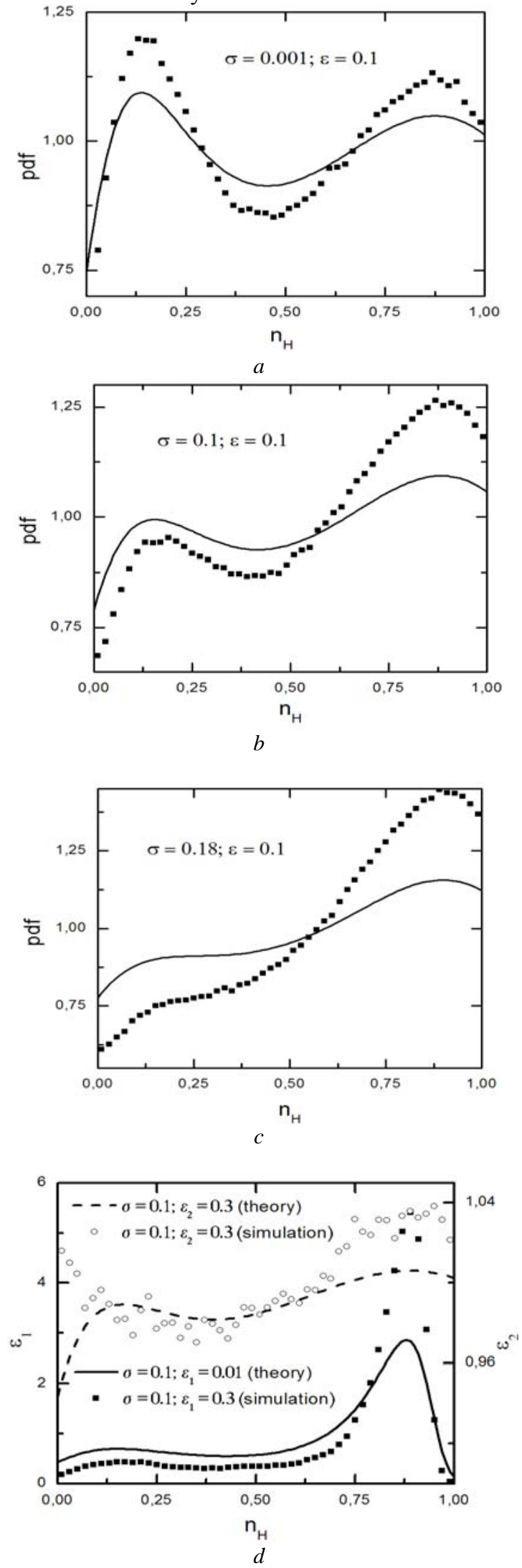

Fig. 2. Additive (a), (b), (c) and multiplicative (d) noise actions on the probability density function distribution. Simulation was carried out for 100 trajectories and 50000 time steps for each trajectory.

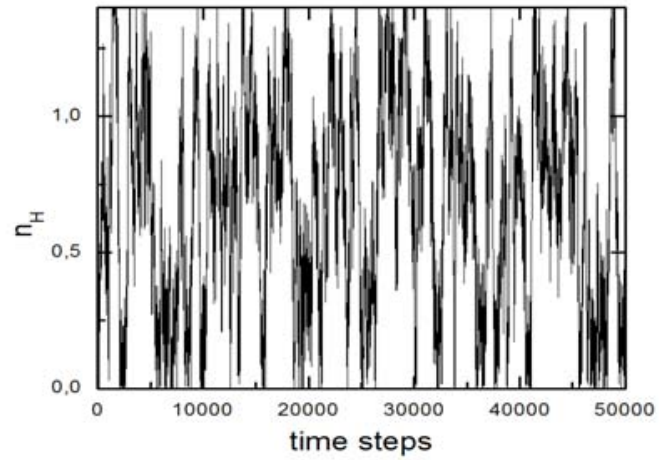

$a$

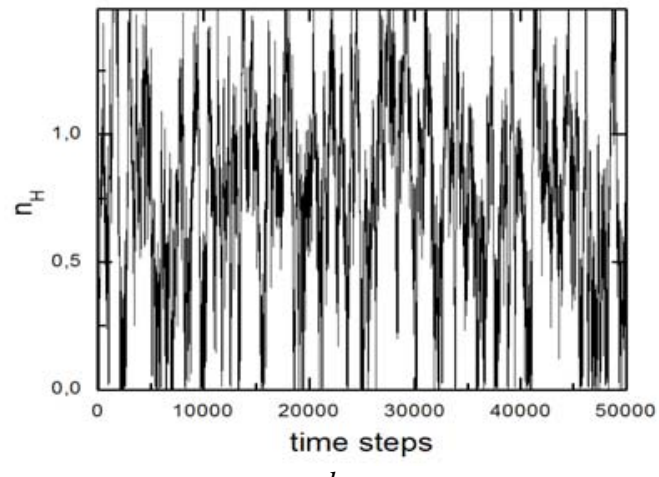

$b$

Fig. 3. The examples of stochastic trajectories for multiplicative noise $\sigma=0.001$ (a) and $\sigma=0.18$ (b). The values of additive noise in both cases have the same magnitude $\varepsilon=0.1$.

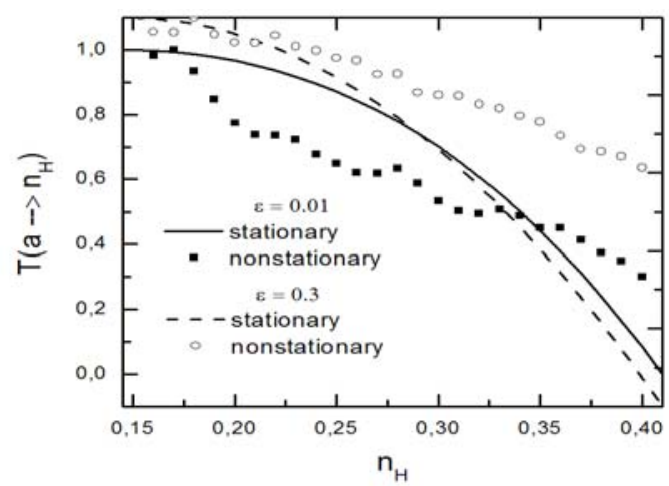

Fig. 4. Normalized MFPT for given values of the additive noise intensity. The value of multiplicative noise is constant $(\sigma=0.1)$.

MFPT in Fig. 4 is corresponded by the bistable potential in Fig. 1c with a reflecting boundary condition at the point $a=0.16$, and absorption boundary condition at the point $b=0.4$. These points indicate the range of metastable state. Given in Table 1 are the values of MFPT for the stationary case. From the type of change

(C) 2010, V. Lashkaryov Institute of Semiconductor Physics, National Academy of Sciences of Ukraine 
in values in Table 1, we can conclude that the increase in the additive noise intensity decreases the lifetime of metastable states. This fact is not favorable for manufacturing data storage systems.

Table 1. Computed stationary values of $T\left(a \rightarrow n_{H}\right)$.

\begin{tabular}{|c|c|c|}
\hline$n_{H}$ & $\begin{array}{c}T\left(a \rightarrow n_{H}\right) \text { for } \\
\varepsilon=0.01\end{array}$ & $\begin{array}{c}T\left(a \rightarrow n_{H}\right) \text { for } \\
\varepsilon=0.3\end{array}$ \\
\hline 0.16 & 690.85 & 39.47 \\
\hline 0.2 & 671.03 & 38.02 \\
\hline 0.3 & 501.91 & 27.1 \\
\hline 0.4 & 109.95 & 5.58 \\
\hline
\end{tabular}

\section{Concluding remarks}

Using the Fokker-Planck equation and its solutions, we studied the noise-dependence properties of the critical behavior of the spin-crossover compound based on extended phenomenological model for spin-crossover compound kinetics in the noise case action. The investigated bistable range may be induced by light irradiation that leads to occurrence of light-induced optical hysteresis. Non-equilibrium dynamical investigation in terms of heat bath firstly developed in [12] has been extended here to the case of two noises action. Dynamical study of the presented noisy model has been made in the frame of Heun algorithm also known as explicit trapezoidal method. The probability density function distribution for stationary and nonstationary dynamics has been obtained. Also, we have shown that the multiplicative noise influence may lead to transition from the metastable to stable state. Action of additive noise is reduced to suppression of the potential barrier height. Increasing the noise intensity provokes a decrease in the decay time of metastable states.

\section{References}

1. Spin Crossover in Transition Metal Compounds 1, 2, 3, Eds. P. Gütlich, H.A. Goodwin. SpringerVerlag, Berlin, 2004.

2. J.A. Real, A.B. Gaspar, M.C. Muñoz, P. Gütlich, V. Ksenofontov and H. Spiering, Bipyrimidinebridged dinuclear iron(II) spin crossover compounds, in: Spin Crossover in Transition Metal Compounds 1, Eds. P. Gütlich, H.A. Goodwin, p. 167-193. Springer-Verlag, Berlin, 2004.

3. S. Decurtins, P. Gütlich, C.P. Köhler, H. Spiering and A. Hauser, Light-induced excited spin state trapping in a transition-metal complex: The hexa-1-propyltetrazole-iron (II) tetrafluoroborate spin-crossover system // Chem. Phys. Lett. 105(1), p. 1-4 (1984).

4. A. Desaix, O. Roubeau, J. Jeftic, J.G. Haasnoot, K. Boukheddaden, E. Codjovi, J. Linarès, M. Noguès, and F. Varret, Light-induced bistability in spin transition solids leading to thermal and optical hysteresis // Europ. Phys. J. B 6(2), p. 183-193 (1998).
5. C. Enachescu, R. Tanasa, A. Stancu, G. Chastanet, J.-F. Létard, J. Linares, and F. Varret, Ratedependent light-induced thermal hysteresis of [Fe(PM-BiA)2(NCS)2] spin transition complex // J. Appl. Phys. 99(8), 08J504 (2006).

6. Iu.V. Gudyma, A.Iu. Maksymov, Theoretical analysis of the states of spin-crossover solids under cross-correlated noises // Physica B 405(11), p. 2534-2537 (2010).

7. L.S. Pontryagin, A.A. Andronov, A.A. Vitt // Zhurnal Eksp. Theor. Fiziki 3, p. 165 (1933) (in Russian); English transl. in: Noise in Nonlinear Dynamical Systems, Eds. F. Moss and P.V.E. McClintock. Cambridge University Press, Cambridge, 1989.

8. A. Hauser, P. Gutlich and H. Spiering, High-spin $\rightarrow$ low-spin relaxation kinetics and cooperative effects in the $\left[\mathrm{Fe}(\mathrm{ptz})_{6}\right]\left(\mathrm{BF}_{4}\right)_{2}$ and $\left[\mathrm{Zn}_{1-\mathrm{x}} \mathrm{Fe}_{\mathrm{x}}(\mathrm{ptz})_{6}\right]\left(\mathrm{BF}_{4}\right)_{2}$ $(\mathrm{ptz}=1$-propyltetrazole) spin-crossover systems // Inorg. Chem. 25(23), p. 4245-4248 (1986).

9. A. Hauser, Intersystem crossing in the $\left[\mathrm{Fe}(\mathrm{ptz})_{6}\right]\left(\mathrm{BF}_{4}\right)_{2}$ spin crossover system $(\mathrm{ptz}=1$ propyltetrazole) // J. Chem. Phys. 94(4), p. 27412749 (1991).

10. A. Hauser, Cooperative effects on the $\mathrm{HS} \rightarrow \mathrm{LS}$ relaxation in the $\left[\mathrm{Fe}(\mathrm{ptz})_{6}\right]\left(\mathrm{BF}_{4}\right)_{2}$ spin-crossover system // Chem. Phys. Lett. 192(1), p. 65-70 (1992).

11. F. Varret, K. Boukheddaden, J. Jeftic and O. Roubeau, A macroscopic approach to the lightinduced instability of cooperative photo-switchable systems // Mol. Cryst. Liquid Cryst. 335, p. 561572 (1999).

12. Yu. Gudyma and O. Semenko, Non-equilibrium kinetics in spin-crossover compounds // Phys. status solidi (b) 241(2), p. 370-376 (2004).

13. C. Enachescu, J. Linarès and F. Varret, Comparison of static and light-induced thermal hysteresis of a spin-crossover solid, in a mean-field approach // J. Phys.: Condens. Matter 13(11), p. 2481-2497 (2001).

14. Yu. Gudyma and B. Ivans'kii, Behavior of asymmetric bistable system under influence of cross-correlated noises // Mod. Phys. Lett. B 20(20), p. 1233-1239 (2005).

15. N.G. van Kampen, Stochastic Processes in Physics and Chemistry. North-Holland, Amsterdam, 1980.

16. D.-j. Wu, L. Cao, S.-z. Ke, Bistable kinetic model driven by correlated noises: Steady-state analysis // Phys. Rev. E 50(4), p. 2496-2502 (1994).

17. M.S. Miguel and R. Toral, Stochastic effects in physical systems, in: Instabilities and Nonequilibrium Structures VI, Eds. E. Tirapegui, J. Martínez and R. Tiemann, p. 35-130. Kluwer Academic Publ., Dordrecht, 2000.

18. U.M. Ascher and L.R. Petzold, Computer Methods for Ordinary Differential Equations and Differential Algebraic Equations. SIAM, Philadelphia, 1998. 
19. P. Hanggi, P. Talkner and M. Borkovec, Reactionrate theory: fifty years after Kramers // Rev. Mod. Phys. 62(2), p. 251-341 (1990).

20. Iu. Gudyma, A. Maksymov, and C. Enachescu, Decay of a metastable high-spin state in spincrossover compounds: mean first passage time analysis // Europ. Phys. J. B (2010), (in press). 